Aufklärung und Skepsis 


\section{Aufklärung \\ und Skepsis}

Internationaler Heine-Kongreß 1997

zum 200. Geburtstag

Herausgegeben von Joseph A. Kruse,

Bernd Witte und Karin Füllner

Verlag J. B. Metzler

Stuttgart . Weimar 


\section{Redaktion:}

Bernd Füllner, Karin Füllner

Die Deutsche Bibliothek - CIP-Einheitsaufnahme

Aufklärung und Skepsis / Internationaler Heine-Kongreß 1997 zum 200. Geburtstag / hrsg. von Joseph A. Kruse ...

- Stuttgart ; Weimar : Metzler, 1998

ISBN 978-3-476-01621-8

ISBN 978-3-476-01621-8

ISBN 978-3-476-03751-0 (eBook)

DOI 10.1007/978-3-476-03751-0

Dieses Werk einschließlich aller seiner Teile ist urheberrechtlich geschützt. Jede Verwertung außerhalb der engen Grenzen des Urheberrechtsgesetzes ist ohne Zustimmung des Verlages unzulässig und strafbar. Das gilt insbesondere für Vervielfältigungen, Übersetzungen, Mikroverfilmungen und die Einspeicherung und Verarbeitung in elektronischen Systemen.

(C) 1999 Springer-Verlag GmbH Deutschland

Ursprünglich erschienen bei J. B. Metzlersche Verlagsbuchhandlung und Carl Ernst Poeschel Verlag GmbH in Stuttgart 1999 


\section{Vorwort}

Karin Füllner · Joseph A. Kruse · Bernd Witte..................................... XI

Rede zur Eröffnung des Internationalen Heine-Kongresses zum 200. Geburtstag des Dichters in Düsseldorf

Wolf Biermann: Heine, unsere Zuckererbsen.

I. Diskurse der Aufklärung............................................................ 19

Peter Bürger: Zweite Aufklärung. Ein Versuch über Heine...................... 19

Gerhard Höhn: „Wissenschaft der Freiheit« und jesuitische

Falschmünzerei. Zu Heines Politikbegriff.

Gerhardt Stenger: Zur Entstehungsgeschichte des französischen

Materialismus im Zeitalter der Aufklärung (nebst kritischen Anmerkungen zu Heines »Zur Geschichte der Religion und Philosophie in Deutschland «)

Manfred Windfuhr: Heine als Polemiker............................................... 57

Jan-Christoph Hauschild: Die Wunden Heines...................................... 71

Rainer Kolk: Über die Aufgabe des Geschichtsschreibers. Heines "Ludwig Börne. Eine Denkschrift« im Kontext

Michael Hofmann: Veranschaulichung von Ambivalenz in Bildern des Tanzes. Dichotomien der Aufklärung und ihre poetische Bearbeitung bei Heine und Wieland.

II. Deutsche Nation und europäische Nationen

Wolfgang J. Mommsen: Heinrich Heine und die Deutschen.

Stefan Bodo Würffel: Geistige Bastillen und Tempel der Freiheit. Zur Konstruktion politischer Identität bei Heine im Spannungsfeld von Nationalismus und Kosmopolitismus.

Waltraud Maierhofer: Italia und Germania. Zum Frauen- und

Deutschlandbild in Heines italienischen "Reisebildern«.

Renate Stauf: »...es giebt jetzt in Europa keine Nazionen mehr, sondern nur Partheyen«. Heines Europa im Widerstreit von Geschichte und Utopie

III. Judenemanzipation als »Probierstein« der Aufklärung.

Moshe Zimmermann: Von der Verbrennung von Büchern und Menschen. 
William W. Hagen: Von »heidnischer Nazionalität« zu »christlicher

Fraternität« und »allgemeiner Völkerliebe«. Heines Überlegungen

zur polnischen Frage und zum europäischen Nationalismus..

Norbert Waszek: Aufklärung, Hegelianismus und Judentum im Lichte der Freundschaft von Heine und Gans.....

Gunnar Och: „Schalet, schöner Götterfunken« - Heinrich Heine und die jüdische Küche.

Hiroshi Kiba: Aufklärung und Ethnizität bei Heinrich Heine.

Peter A. Schmid: Deutschtum und Judentum bei Hermann Cohen und Heinrich Heine.

Michael Perraudin: Irrationalismus und jüdisches Schicksal.

Die thematischen Zusammenhänge von Heines "Ideen. Das Buch Le Grand«.....

Hartmut Steinecke: „Wir stammen von Schlemihl«. Jüdische Dichter-

Bilder in Heines Spätwerk von Jehuda ben Halevy bis

Rabbi Faibisch.

Jonathan Skolnik: Die seltsame Karriere der Familie Abarbanel.

Anne Maximiliane Jäger: Bacherach - Frankfurt - Toledo. Heines

»Rabbi von Bacherach« als literarisches Projekt der jüdischen Aufklärung.

IV. Historisierung des Mythos, Mythisierung der Aktualität......

Ernst Behler: Mythos und Ironie im literarischen Diskurs

Heinrich Heines.

Andreas Böhn: Der fremde Mythos und die Mythisierung des Fremden.

Heines politisch-literarische Mythologie in "Vitzliputzli«...

Markus Winkler: Heines Napoleon-Mythos.

Wulf Wülfing: Luise gegen Napoleon, Napoleon gegen Barbarossa.

$\mathrm{Zu}$ einigen Positionen Heines in einem Jahrhundert der

Mythenkonkurrenzen.

Cornelia Epping-Jäger: Mythos Paris? - Heinrich Heines

daguerreotypische Schreibart.

George F. Peters: Der Dichter als Mythenbeleber. Goethe, Heine und Faust.

Olaf Briese: Venus - Madonna - Maria. Über Heines

Marienverständnis.

V. Autonome versus politische Literatur.

Jakob Hessing: Auf Tod und Leben. Heinrich Heine zwischen autonomer und politischer Literatur.

Takanori Teraoka: Verteidigung des ıSchönenc. Plädoyer für Kunstautonomie in Heines Brief an Gutzkow. 
Sabine Bierwirth: Tambour und Trommler. Heines Versuch einer Synthese >politisch-romantischer/ Dichtung .....

Walter Erhart: Heinrich Heine: Das Ende der Geschichte und "verschiedenartige« Theorien zur Literatur

VI. Menschheitsideal und Menschenrechte.

Sigrid Weigel: „Das Wort wird Fleisch, und das Fleisch blutet«Heines Reflexion der Menschenrechte im Buch Gottes und in der Weltgeschichte.

Franz Schüppen: Heinrich Heine und die Abschaffung der Armut.Ein deutscher Dichter in der Welt des französischen Bürgerkönigs

Terence M. Holmes: Welcher Gedanke geht wessen Tat voraus?

Zur Revolutionsproblematik bei Heine.

VII. Philosophie, Religion, Psychologie im Werk Heines.

Ulrich Stadler: Heines »Nordsee III" als programmatischer Beitrag zur $\gg$ Neuen Mythologie`.

Willi Goetschel: Heines Spinoza: Ent/Mythologisierung der Philosophie als Projekt der Entzauberung und Emanzipation.

Jürgen Ferner: Theatrum historiae: Anmerkungen zur Mäuseparabel in »Shakspeares Mädchen und Frauen«.

Christian Liedtke: »Das Leben ist weder Zweck noch Mittel; das

Leben ist ein Recht«. Heines Kritik des teleologischen Denkens.

Ortwin Lämke: Heines »Geschichtsschreibung der Gegenwart«.

Zu Artikel VI der »Französischen Zustände«.

Kai Neubauer: Freiheit, Ironie, Sinnlichkeit: Heine und Bruno

VIII. Die internationale Heine-Rezeption.

Ariane Neuhaus-Koch: »Heine hat alle Stadien der Emigration mit uns geteilt«. Aspekte der Exilrezeption 1933-1945.

Dieter Schiller: Heinrich Heine als Leitfigur in der Exilpublizistik 1933-1945

Alfred Opitz: Von Heine bis Baudelaire. Zum Begriff der »literarischen Race« bei Hermann Bahr.

Manuela Delille: Die Heine-Rezeption in Portugal. Von der Romantik bis zum Ersten Weltkrieg.

Arno Gimber: Von Perlen, Mond und Wasserfrauen. Die Heine-

Rezeption in der spanischen Literatur der Jahrhundertwende.

Werner Wögerbauer: Der krumme Leser. Heinrich Heine und

Paul Celan.

Su-Yong Kim: Die »Loreley« in Korea. Eine Rezeptionsgeschichte 
Zhang Yushu: Das Heine-Bild in China.

Ma Wentao: Heines Gedichte im Deutschstudium in China.

IX. Der Dichter Heine.

Fritz Mende: Die »Wahrheit des Gefühls«. Bemerkungen zu Heines

Selbstverständnis als ein Dichter der Moderne

Mauro Ponzi: Heine und die neue Zeit oder Die schwarze Sonne der Zukunft

Lucien Calvié: "Aus den Memoiren des Herren von Schnabelewopski«.

Autobiographie, Parodie, Kunstperiode und Politik

Albrecht Betz: Der letzte Sommernachtstraum. Heines Gedicht "An die Mouche«.

Paul Peters: Heine als Plebejer.

Bettina Knauer: Heinrich Heines »Florentinische Nächte«. Form und

Funktion novellistischen Erzählens und esoterischer Allegorik.

Beate Perrey: Rationalisierung von Sinnlichkeit in Heines »Lyrischem

Intermezzo«. Das »Hohelied» als poetisches Modell im

Zerrspiegel »kleiner maliziöser Lieder«....

Louis Hay: Notizen zu den »Notizen« oder Heine als Schriftsteller.

Ralf Schnell: Heines Essayismus.

Sonderveranstaltung

Manfred Windfuhr: Rückschau und Bilanz. Zum Editionsprofil der

Düsseldorfer Heine-Ausgabe.

Öffentliche Vorträge im Düsseldorfer Malkasten

Odo Marquard: Skepsis in der Moderne. Überlegungen im Blick auf Heinrich Heine.

Jacques Revel: Heine zwischen Deutschland und Frankreich.

Eine historische Vergewisserung.

Georges-Arthur Goldschmidt: Heine, ein deutscher Dichter

in Frankreich

Verzeichnis der Autorinnen und Autoren 


\section{Siglen}

\section{Heinrich Heines Werke und Briefe}

B

Heinrich Heine: Sämtliche Schriften, hrsg. von Klaus Briegleb. München 1968-1976

DHA

Heinrich Heine: Historisch-kritische Gesamtausgabe der Werke. (Düsseldorfer Ausgabe). In Verbindung mit dem Heinrich-Heine-Institut hrsg. von Manfred Windfuhr. Hamburg 1973-1997

HSA

Heinrich Heine: Werke, Briefe, Lebenszeugnisse. Säkularausgabe, hrsg. von den Nationalen Forschungs- und Gedenkstätten der klassischen deutschen Literatur in Weimar (seit 1991: Stiftung Weimarer Klassik) und dem Centre National de la Recherche Scientifique in Paris. Berlin und Paris 1970ff.

\section{Weitere Abkürzungen}

Galley/Estermann Heinrich Heines Werk im Urteil seiner Zeitgenossen, hrsg. von Eberhard Galley und Alfred Estermann. Hamburg 1981ff.

HJb Heine-Jahrbuch, hrsg. vom Heinrich-Heine-Institut Düsseldorf. Hamburg 1962-1994; Stuttgart, Weimar 1995ff.

Höhn: Heine-

Handbuch Gerhard Höhn: Heine-Handbuch. Zeit, Person, Werk. Stuttgart 1987

Höhn: Heine-

Handbuch ${ }^{2} 1997$ Gerhard Höhn: Heine-Handbuch. Zeit, Person, Werk. 2. aktualisierte und erweiterte Aufl. Stuttgart, Weimar 1997

Werner/Houben Begegnungen mit Heine. Berichte der Zeitgenossen, hrsg. von Michael Werner in Fortführung von H.H. Houbens »Gespräche mit Heine«. 2 Bde. Hamburg 1973 


\section{Vorwort}

Kolloquien, Symposien und Kongresse scheinen sich inzwischen auch innerhalb der Germanistik gelegentlich zu überschlagen. Die im gebührenden Abstand meistens mit etwas Verzögerung - herausgebenen Sammelbände, die das Ergebnis in Gestalt sämtlicher Vorträge enthalten, versuchen das Resümee solcher Tage für die spätere wissenschaftliche Debatte zu bündeln und sind glücklicherweise oft genug für die nachfolgende Forschung anregend und wichtig. Die Fülle von Anlässen mit ihren Gepflogenheiten von Zusammenkünften und späterer Drucklegung mag man unter der Hand als einen der neuen, mittlerweile überhaupt nicht mehr als zwanglos empfundenen Riten und sich verselbständigenden Automatismen belächeln. $\mathrm{Ab}$ und an erzählen solche Bücher dann aber doch mehr, als in den Beiträgen gesagt wird. Für den Düsseldorfer Heine-Kongre $\beta$ aus Anlaß des 200. Geburtstages des Dichters gilt das jedenfalls ganz besonders. Der Kongre $\beta$ selbst und in dessen Folge der vorliegende Kongreßband bilden zusammengenommen tatsächlich, gerade angesichts der inzwischen verstrichenen Zeit, ohne Übertreibung eine geradezu wunderbar zu nennende Überraschung innerhalb der Geschichte der Heine-Rezeption mit ihren wahrlich nicht immer für die Nachwirkung des Dichters günstigen Phasen. Mit diesem - wie auch immer im einzelnen zu charakterisierenden - Echo auf den Kongreß-Plan und mit diesem Erfolg des Kongresses selbst, konnten die Veranstalter bei allem guten Willen nicht ohne weiteres rechnen.

Der Anla $ß$ für einen großangelegten Heine-Kongreß war mit dem 200. Geburtstag des Schriftstellers allemal gegeben. Das Heine-Jahr 1997 entwickelte sich dann bereits vor dessen Beginn Ende Mai 1997 mit seinen durchaus beachtlichen internationalen Aktivitäten zu einem überwältigenden Kulturereignis weit über Düsseldorf hinaus. In den verschiedensten Medien fand Heine immer wieder Platz, wurde das Heine-Jahr kommentiert oder provozierte eigene Auseinandersetzungen und Arbeiten in Fernsehen und Rundfunk, als Spektakel oder bescheidenes Gedenken, im Musikleben und auf der Theaterbühne. Manchen Mitgliedern zumal der deutschen Heine-Gemeinde schien es im atemlosen Wettlauf der HeineTermine gar notwendig, den Autor vor der unermeßlichen Flut an plötzlich zutage getretenem Interesse in Schutz zu nehmen. Dem von der Heinrich-Heine-Universität und dem Heinrich-Heine-Institut in Düsseldorf gemeinsam ausgetragenen Kongreß fält daher vor allem im Nachhinein angesichts der als so erfolgreich und spektakulär zu wertenden Heine-Ausstellung des Heinrich-Heine-Instituts, die in Düsseldorf und Paris gezeigt wurde, aber auch neben anderen Ereignissen wie 
beispielsweise der gewissermaßen als politisches Bekenntnis zu wertenden Großveranstaltung in der Deutschen Oper am Rhein am 13. Dezember 1997, also am Heine-Geburtstag selbst, die vor allem durch die Ansprache von Bundespräsident Roman Herzog ausgezeichnet wurde, eine das überwältigende Publikumsinteresse begleitende Funktion kritischen Nachdenkens und wissenschaftlichen Resümees zu. - Und das unter Berücksichtigung der Tatsache, daß der HeineKongre $\beta$ in seiner Größenordnung selber eine dem gesamten Jahr entsprechende Dimension erhalten hat, die man ansonsten innerhalb der Literaturwissenschaft nur mit den jährlichen Goethe-Tagungen in Weimar vergleichen kann. Insofern darf nicht gleich bei solch bewundernswert intensivem Interesse an Heine von übertriebenen literaturwissenschaftlichen Ansprüchen oder gigantomanischen Absichten gesprochen werden, sondern nur von einer existentiell begründeten Anteilnahme, der offenbar das Heinesche Werk, seine Persönlichkeit und seine Wirkung zweifellos den ansprechendsten Gegenstand boten und die sich in wissenschaftlichen Zusammenkünften von Fall zu Fall angemessen ausdrücken muß.

Der erste große internationale Heine-Kongreß hatte vor einem Vierteljahrhundert zum 175. Geburtstag des Dichters in Düsseldorf stattgefunden und sein wenig später ausgerichtetes Parallelereignis in Weimar und Berlin (Ost) besessen. Beide Veranstaltungen sind in Sammelbänden dokumentiert. Davor bestand das HeineGedenken neben und trotz aller massiven Denkmalstreitigkeiten und angesichts einer ganzen Verfolgungsgeschichte, wodurch Heines Nachwirkung wesentlich geprägt worden ist, eher in Festakademien, Ausstellungen oder publizistischen Beiträgen auch und gerade seiner Befürworter. Das gilt selbst für die Exilliteratur in unserem Jahrhundert, wie einige Beiträge in diesem Band eindrucksvoll belegen. Das 100. Todesjahr 1956 bildete dann immerhin den Ausgangspunkt für eine anfangs noch gemeinsam geplante deutsch-deutsche Heine-Ausgabe, deren bibliographische und dokumentarische Vorarbeiten sich schließlich zu zwei getrennten respektablen und erfolgreichen Unternehmen entwickelten. In Düsseldorf wurde im selben Jahr die bestehende Heine-Sammlung um den handschriftlichen Nachla $\beta$ des Dichters in der damaligen Landes- und Stadtbibliothek bereichert. Gleichzeitig beinhaltete das Gedenken zum 100. Todestag des Dichters das Gründungsdatum der Heinrich-Heine-Gesellschaft, die sich in der Folge für die Nachwirkung in der Öffentlichkeit ungemein verdient gemacht hat. Die Verselbständigung der Neueren Handschriftenabteilung der alten Bibliothek mit dem HeineArchiv im Jahre 1970 als Heinrich-Heine-Institut der Landeshauptstadt Düsseldorf und die sehr viel später endlich stattgefundene Benennung der Düsseldorfer Universität nach Heinrich Heine im Jahre 1988/89 trugen das ihrige zu einer Akzeptanz des Dichters an seinem Geburtsort bei. Zum 125. Todestag 1981 wurde in Düsseldorf übrigens von seiten des Heinrich-Heine-Instituts und der HeinrichHeine-Gesellschaft ein Kolloquium zum sspäten Heiner veranstaltet, das bereits ein entspannteres und gleichzeitig differenzierteres Verhältnis zu den Problemen 
der Heine-Philologie und der Heine-Rezeption konstatieren konnte. Auch diese Ergebnisse wurden in einem eigenen Band der »Heine-Studien « festgehalten. Somit waren glücklicherweise auf der einen Seite Ausgangspunkt und Summe und auf der anderen Seite Wege der Spezifizierung wie Spezialisierung gewonnen, die jeweils der umsichtigen Pflege und erneuten Auseinandersetzung bedurften. Die passendste Gelegenheit hierzu bot sich aus Anlaß der Universitätsbenennung bei einem ganz von Düsseldorfer Heine-Fachleuten gestalteten Heine-Symposium der Universität im Juni 1989, das sich des Autors »im Spannungsfeld von Literatur und Wissenschaft" annahm. Der Abschluß der Arbeiten an der Düsseldorfer Historisch-kritischen Heine-Ausgabe (DHA), eine materielle Glanzleistung der Landeshauptstadt Düsseldorf, besiegelt durch das Erscheinen des letzten und 16. Bandes mit Nachträgen und Register just zum Beginn des Heine-Jahres 1997, symbolisierte schließlich den Düsseldorfer Fortschritt beim Andenken an den Dichter auf eindringliche Weise.

Der Internationale Heine-Kongreß in Düsseldorf vom 25. bis 30. Mai 1997 stellte deutlich und sichtbar einen wesentlichen Teil des Gesamtprogramms zum HeineJahr 1997 dar, das von der Landeshauptstadt Düsseldorf, der Heinrich-HeineGesellschaft und der Heinrich-Heine-Universität Düsseldorf ausgerichtet wurde. Das Kuratorium des Heine-Jahres hatte sich unter Federführung der Düsseldorfer Oberbürgermeisterin Marlies Smeets von seiten der Stiftungen, der Wirtschaft, der Banken und der öffentlichen Hand auf effektive Weise zusammengetan. Die Vorsitzende der Heinrich-Heine-Gesellschaft, Johanna von Bennigsen-Foerder, leistete dabei gerade zur Bündelung des privaten Engagements ihren außerordentlichen Beitrag. Der Rektor der Heinrich-Heine-Universität, Gert Kaiser, trug von vornherein den Gedanken des Heine-Jahres und des Kongresses mit, so daß der dafür zuständige und beauftragte Bereich der Neueren Germanistik zusammen mit dem Heinrich-Heine-Institut, das darüber hinaus an den vielfältigsten Projekten des Heine-Jahres beteiligt war, die Planung beginnen konnte. Der Kongreß im Rahmen des Heine-Jahres wurde dankenswerterweise gefördert durch die Heinrich-Heine-Universität, die Stadt Düsseldorf, das Ministerium für Wissenschaft und Forschung des Landes Nordrhein-Westfalen und die Deutsche Forschungsgemeinschaft, die für die finanziellen Voraussetzungen Sorge trugen. Zur Vorbereitung des Düsseldorfer Internationalen Heine-Kongresses 1997 konnte ein wissenschaftlicher Beirat gewonnen werden, der in anregender und einvernehmlicher Weise über Inhalte und Teilnahme die notwendigen Entschließungen traf: Walter Hinck (Köln), Bernd Kortländer (Düsseldorf), Renate Schlesier (Paderborn), Michael Werner (Paris) und Manfred Windfuhr (Düsseldorf) ist für Rat und Tat herzlich zu danken.

Als Überschrift für den Internationalen Heine-Kongreß wurde die bereits inhaltlich anspielungsreiche Formel gewählt: »Aufklärung und Skepsis. Heinrich Heine 
zum 200. Geburtstag«. Damit sollte einerseits jene deutsche und europäische Tradition mit ihrem Vernunfts- wie Zukunftsglauben in den Blick kommen, der Heines Schreiben in der Hauptsache verpflichtet ist, andererseits neben der Aufklärung im romantischen Zeitalter die Heineschen Leistungen von Vorbehalt und Brechung, Ästhetik und Engagement, aber selbstverständlich auch die Bedingungen seiner jüdischen Herkunft und Forderungen nach Emanzipation und Humanität thematisiert werden. Das Bild des Autors der Moderne sollte auch bei Gelegenheit eines solchen Kongresses Gegenstand unterschiedlichster Annäherungen sein. Neun Sektionen steckten den Rahmen ab, die nunmehr auch die Kapitelüberschriften des Kongreßbandes bilden. Die kritische Vergegenwärtigung aufklärerischer Ansprüche und Tendenzen unter der Überschrift »Diskurse der Aufklärung" eröffnete die Fragestellung, die dann um die Betrachtung des Phänomens »Deutsche Nation und europäische Nationen« erweitert wurde, während darauf aufbauend die "Judenemanzipation als >Probierstein` der Aufklärung" zu erörtern war. Die vierte Sektion wurde als »Historisierung des Mythos, Mythisierung der Aktualität« überschrieben, der sich der Komplex "Autonome versus politische Literatur" anschloß. Mit dem Thema "Menschheitsideal und Menschenrechte« wurde der Übergang geschaffen zur siebten Sektion »Philosophie, Religion, Psychologie im Werk Heines«. In der achten Sektion wurde »Die internationale Heine-Rezeption« analysiert, während die letzte Sektion, die schlicht und einfach »Der Dichter Heine« überschrieben war, abschließend nach den poetischen Leistungen des Autors Heine fragte. Ergänzt wurden die Sektionen durch eine Sonderveranstaltung zum Editionsprofil der Düsseldorfer Heine-Ausgabe und durch drei Abendvorträge, die im Unterschied zu den übrigen Vorlesungen und Referaten, die in den Hörsälen der Heinrich-Heinrich-Heine-Universität stattfanden, im Haus des Künstlervereins Düsseldorfer Malkasten - gelegen im Park der Brüder Jacobi - gehalten wurden, wo bereits der Kongreß von 1972 getagt hatte.

Auf die Ausschreibung waren mehr Anmeldungen für Vorträge erfolgt, als überhaupt im zur Verfügung stehenden Zeitraum unterzubringen waren. Über sechzig Referate konnten schließlich eingeplant werden, die von etwa vierzig HeineExpertinnen und -Experten moderiert wurden. Insgesamt waren Beiträgerinnen und Beiträger aus fünfzehn Ländern vertreten. Darunter befanden sich neben Literaturwissenschaftlern auch Philosophen, Historiker und Sozialwissenschaftler. Weitere 500 Anmeldungen zum Kongreß ergaben zusammen mit der Expertengruppe eine höchst interessierte und bunte Versammlung zugunsten einer äußerst lebhaften Tagung. Das Besondere bildete auf dem Kongreß die von vielen Seiten und häufig auch nachträglich eigens lobend hervorgehobene positive Atmosphäre, die die ursprüngliche Konzeption bestätigte und einer zwanglosen Mischung der Heine-Forscher verschiedenster Provenienz zu verdanken war. Gerade die zahlreichen öffentlichen Diskussionen und privaten Gespräche zwischen älteren und 
jüngeren Heineforscherinnen und -forschern wurden als ausgesprochen anregend für die weitere Arbeit erfahren. Insofern hat der Internationale Heine-Kongreß 1997 in Düsseldorf zweifellos neue Anregungen für die Forschung gegeben. Die politische Vereinnahmung Heines, die noch 1972 zu starken Parteiungen führte, wurde sehr viel differenzierter diskutiert, das jüdische Thema bildete, wie bisher noch nie, einen Schwerpunkt des Kongresses, und die Frage nach »Aufklärung und Skepsis", zugespitzt in Peter Bürgers Plädoyer für eine "zweite" skeptische Aufklärung, wird für die Heineforschung impulsgebebend sein können.

Eine gelungene >Heinesche Rheinfahrt zur Loreley` beschloß am Freitag, dem 30. Mai 1997, über das Besichtigungs- und Erlebnisprogramm hinaus, das in die Heine-Ausstellung »Ich Narr des Glücks« in der Düsseldorfer Kunsthalle und in die Heine-Sonderausstellung der Universitäts- und Landesbibliothek über die von Geldern-Haggadah und Heines »Der Rabbi von Bacherach" geführt, einen Empfang der Oberbürgermeisterin im Rathaus und ein festliches Abendessen im Malkasten auf Einladung der Düsseldorfer Industriellenfamilie Henkel enthalten hatte, einen Heine-Kongre $\beta$ von besonders debattierfreudiger, aber friedvoller Tönung und trotz seiner Größe dennoch immer auch intimer Färbung. Der freundlichironische Abschluß durch eine Rheinreise erinnerte seinerseits an einen als fulminant empfundenen, von privatem Ernst grundierten Beginn.

Denn mit dem Vortrag Wolf Biermanns »Die Loreley stürzt in den Rhein / Damit sie nicht singen muß«, hier unter einem anderen Titel veröffentlicht, wurde der Internationale Heine-Kongreß am Sonntag, dem 25. Mai 1997, im vollbesetzten Düsseldorfer Schauspielhaus eröffnet. Der Heine-Preisträger der Stadt Düsseldorf (1993) und Heine-Gastprofessor der Universität (1994/95) sprach und sang, ausgehend vom Versepos "Deutschland. Ein Wintermährchen", dem er im Jahre 1972 seine eigene deutsch-deutsche Fassung an die Seite gestellt hatte, über das Verhältnis von Politik und Poesie im Werk Heines. Die Kongreßvorträge in der Universität wurden am Montag, dem 26. Mai, eingeleitet durch Hauptvorträge zu den sechs ersten Sektionen. Peter Bürger (Bremen) führte mit seinem Beitrag "Die Aufklärung mit Heine lesen" unmittelbar in das Zentrum des Kongreßthemas »Aufklärung und Skepsis« ein, indem er, wie oben angemerkt, mit Heine für eine "zweite" skeptische Aufklärung plädierte, die als Aufhebung des ungebrochenen Fortschrittsoptimismus der ersten zu begreifen sei. Heines schwierige Stellung und das widersprüchliche Verhältnis der Deutschen zum Dichter untersuchte der Düsseldorfer Historiker Wolfgang J. Mommsen. Wenn auch die Rezeption heute nicht mehr so polarisierend verlaufe, bleibe Heine ein wunzeitgemäßer Zeitgenosse«, der sich gegen Vereinnahmungen sperre. Der israelische Historiker Moshe Zimmermann (Jerusalem) blickte aus der Perspektive der postemanzipatorischen Lösungen der Judenfrage (»Zionismus einerseits und \Endlösung (andererseits «) kritisch zurück auf die Dialektik der Emanzipationsbestrebungen der Vormärzzeit. Der Polarisierung und dem Zusammenspiel von Mythos 
und Ironie im literarischen Diskurs Heinrich Heines widmete sich der Vortrag von Ernst Behler (Seattle/ Washington), einem der größten Kenner der Romantik, der kurze Zeit nach dem Kongreß, auf dem er nach gerade überstandener Erkrankung wieder positiv in die Zukunft blickte, seinem schweren Leiden erlegen ist. Er sah in affirmativer Mythisierung und skeptischer Ironisierung innerhalb der sprachlichen Gestaltung der Texte das antagonistische Weltbild des Autors gespiegelt. Jakob Hessing (Jerusalem), zeichnete Heines Weg einer Literatur zwischen künstlerischer Autonomie und revolutionärer Verpflichtung nach und kam zu dem Schluß, daß Heine letztlich den Versuch, diesseits aller Transzendenz eine Utopie schon auf Erden zu errichten, abbrach. Der Diskrepanz zwischen dem Kampf um allgemeines Recht und um das Recht des Individuums ging Sigrid Weigel (Zürich) nach, indem sie Heines Position im Blick auf Hannah Arendt, Walter Benjamin und Sigmund Freud diskutierte.

An den folgenden drei Tagen (27.-29. Mai 1997) fanden als Parallelveranstaltungen die Sektionsvorträge statt, die die von den Hauptvorträgen eingeleiteten Themen textbezogen konkretisierten und unter der Leitung von Heine-Expertinnen und -Experten diskutierten.

Dabei stand im Zentrum von Sektion 1 (»Diskurse der Aufklärung«) die Frage nach Heines Politikverständnis im Übergang von französischer Aufklärung zum Frühsozialismus und nach seinem ideengeschichtlichen Ort. Seine Darstellung der französischen Philosophie wurde kritisch beleuchtet, gleichzeitig konnten Parallelen und Übereinstimmungen mit den Ideen von Holbach und Voltaire, Condorcet und Helvétius näher präzisiert werden (Gerhard Höhn, Paris, und Gerhardt Stenger, Nantes). In Vorträgen zu Heines Polemiken (Manfred Windfuhr, Düsseldorf) und zum Verhältnis von Biographie und Werk (Jan-Christoph Hauschild, Düsseldorf) wurde seine Stellung zwischen Romantik und Aufklärung exemplarisch untersucht; im Kongreßband kommt auch die Frage nach seiner >Geschichtswissenschaftı zu Wort (Rainer Kolk, Dresden), ein Vortrag des Kongresses, der dort nicht gehalten werden konnte, wenigstens hier aber dokumentiert ist. Das Tanzmotiv als eine Metapher für die Befreiung der Sinnlichkeit diente abschließend dazu, die Ambivalenz der Aufklärung bei Heine im Vergleich zu Wieland herauszuarbeiten (Michael Hofmann, Bonn).

Die Vorträge der Sektion 2 (»Deutsche Nation und europäische Nationen«) diskutierten Heines Europaidee im Spannungsfeld von Nationalismus und Kosmopolitismus, sozialer Realität und utopischer Erwartung. Das Heine eigene Verfahren negativer Dialektik begründe die Hoffnung auf Aufhebung der Extreme und damit die andauernde Vorbildhaftigkeit und Aktualität seiner Dichtung (Stefan Bodo Würffel, Fribourg, und Renate Stauf, Berlin). Kritisch wurden in diesem Zusammenhang mit einem Blick auf die Kunstgeschichte und die italienischen 
Reisebilder seine Frauenbilder untersucht, die Heine mehrfach in den Kontext seiner Nationenkritik stellt (Waltraud Maierhofer, Iowa City/ Iowa).

Breiten Raum nahm mit neun Vorträgen die Sektion 3 (»Judenemanzipation als >Probierstein ( der Aufklärung () ein und lenkte damit ein besonderes Interesse auf Heines Auseinandersetzung mit dem Judentum. Gefragt wurde nach dem ideengeschichtlichen Bezug zur jüdischen Aufklärung eines Moses Mendelssohn, nach jüdischer Identität bis hin zur jjüdischen Küche`(Gunnar Och, Erlangen), nach Assimilation und Fremdheit (Hiroshi Kiba, Kobe), nach Exil, Leidensschicksal und jüdischer Emanzipation. Eine wichtige Rolle spielte Heines Begegnung mit Eduard Gans und dessen "Verein für Cultur und Wissenschaft der Juden» (Norbert Waszek, Paris). Neben den Schriften »Über Polen« (William W. Hagen, Davis/ California) und »Ideen. Das Buch Le Grand" (Michael Perraudin, Birmingham), dem Nachruf auf "Ludwig Marcus«, aber auch dem Altersgedicht "Jehuda ben Halevy" (Hartmut Steinecke, Paderborn), war der nächstliegende zentrale Text, auf den sich die Vorträge dieser Sektion immer wieder bezogen, Heines Romanfragment "Der Rabbi von Bacherach" (Jonathan Skolnik, New York, und Anne Maximiliane Jäger, Marburg). Damit verbunden war stets die sich bis heute stellende Frage, was vom Dichter als Aufklärer übrigbleibe angesichts der historischen jüdischen Erfahrungen. Das Beispiel Hermann Cohens erlaubte dabei, im Rückblick Hoffnung und Fremdheit thematisieren zu können (Peter A. Schmid, Zürich).

Sektion 4 (»Historisierung des Mythos, Mythisierung der Aktualität«) untersuchte die Besonderheit des Heineschen Stils als Spannung und kritische Wechselwirkung zwischen Mythischem und Ironischem am Beispiel von Heines Napoleonund Barbarossa-Mythos (Markus Winkler,University Park/ Pennsylvania, und Wulf Wülfing, Bochum), am Beispiel seiner Stadtdarstellung (Cornelia EppingJäger, Düsseldorf), im Gedicht »Vitzliputzli« (Andreas Böhn, Mannheim), im Tanzpoem »Der Doktor Faust (George F. Peters, East Lansing/ Michigan) und anhand seines Marienverständnisses (Olaf Briese, Berlin). Dabei wurde Heines spielerischer Umgang mit der Mythologie, sein ironischer Anspruch auf Mythenbildung und -zerstörung konkretisiert und sowohl auf seine politisch-sozialen wie auf seine ästhetischen Funktionen bezogen.

Die Vorträge der Sektion 5 (»Autonome versus politische Literatur«) diskutierten Heines literarisches Selbstverständnis, sein Plädoyer für Kunstautonomie einerseits und sein Eintreten für eine slittérature engagéer als »braver Soldat im Befreyungskriege der Menschheit» andererseits (Takanori Teraoka, Okayama, und Sabine Bierwirth, Athen). Über die Versuche, Heines Position als ambivalente Haltung oder als Synthesebemühung zu verstehen, führte der Ansatz hinaus, in Heines Spätwerk Geschichte als ein Feld vieldeutiger Erzählungen wieder neu zu 
entdecken und somit einen neuen Zusammenhang von Literaturautonomie und Geschichte zu konstituieren (Walter Erhart, Göttingen).

Mit der Revolutionsproblematik bei Heine beschäftigten sich die Vorträge der Sektion 6 (»Menschheitsideal und Menschenrechte«), indem sie vor allem die Bedeutung der französischen Julirevolution in Heines Werken analysierten. Wichtig war in diesem Zusammenhang eine Neudefinition von Heines Zukunftsbild einer deutschen Revolution am Ende von "Zur Geschichte der Religion und Philosophie in Deutschland" (Franz Schüppen, Herne, und Terence M. Holmes, Swansea).

Im besonderen widmete sich Heines Philosophieschrift die Sektion 7 (»Philosophie, Religion, Psychologie im Werk Heines«). Die Frage nach Heines ıSpinozismusı (Willi Goetschel, New York), seine Skepsis gegenüber dem teleologischen Welt- und Menschenbild der aufklärerischen Geschichtsphilosophie (Christian Liedtke, Köln), seine Kritik des hegelschen Geschichtsdenkens, seine Darstellung des Verhältnisses von Natur- und Sozialgeschichte sowie die Beziehung von Sensualismus und Spiritualismus in seinem Werk zumal im Rückbezug auf Bruno (Kai Neubauer, Pisa) wurden darüber hinaus an den Texten $"<$ Verschiedenartige Geschichtsauffassung>«, "Die Nordsee III" (Ulrich Stadler, Zürich), »Französische Zustände" (Ortwin Lämke, Paris), an der Mäuseparabel in "Shakespeares Mädchen und Frauen« (Jürgen Ferner, Essen) und dem späten Gedicht »Beine hat uns zwey gegeben« untersucht.

In Sektion 8 (»Die internationale Heine-Rezeption«) wurde als ein Schwerpunkt die Exilrezeption 1933 bis 1945 vorgestellt. In der Auseinandersetzung mit kultureller Repression und Rassenpolitik im Nationalsozialismus wurde der Autor für viele exilierte deutsche Intellektuelle und Poeten in den Zeitschriften, Anthologien und in der germanistischen Forschung des Exils zur Integrationsfigur (Ariane Neuhaus-Koch, Düsseldorf, und Dieter Schiller, Berlin). In den romanischen Sprachraum führten zwei Vorträge, die sich mit der Rezeption Heines in Portugal (Manuela Delille, Coimbra) und Spanien (Arno Gimber, Amiens) beschäftigten, für die im 19. Jahrhundert in beiden Ländern die Vermittlung durch Frankreich eine große Rolle spielte. Abhängig von den politischen Ereignissen konnten verschiedene Phasen der Rezeption benannt werden. Interessante Einzelstudien stellten die Rückbezüge auf Heine bei Hermann Bahr (Alfred Opitz, Lissabon) und bei Paul Celan (Werner Wögerbauer, Nantes) vor. Ein letzter Themenschwerpunkt dieser Sektion beschäftigte sich mit der fernöstlichen Rezeption: mit dem Heine-Bild in China (Zhang Yushu, Peking), mit Heines Gedichten im chinesischen Deutschstudium (Ma Wentao, Peking) und exemplarisch mit der besonderen Rezeptionsgeschichte des "Loreley«-Gedichtes in Korea (Su-Yong Kim, Seoul). 
Sektion 9 (»Der Dichter Heine«) stellte die Frage nach der Modernität Heines (Fritz Mende, Weimar, und Mauro Ponzi, Rom) und untersuchte in vielen Einzelanalysen den spezifischen Sprachstil bis hin zum raffinierten `Pleberjertum (Paul Peters, Montréal). Dabei rückten auch in den anderen Sektionen sonst weniger beachtete Texte ins Blickfeld: «Aus den Memoiren des Herren von Schnabelewopski« (Lucien Calvié, Grenoble), das Gedicht "Für die Mouche» (Albrecht Betz, Aachen), »Florentinische Nächte" (Bettina Knauer, Erlangen), das »Lyrische Intermezzo" (Beate Perrey, Cambridge), das "Tannhäuserlied», besonders aber die Prosanotizen (Louis Hay, Paris); über den minutiösen Nachweis des Schreibprozesses nach den Autographen wurde der Bogen zu einer neuen Poetikkonzeption geschlagen, nach der Subjektivität und Reflexivität in Heines Sprachstil auf die produktionsästhetisch orientierten Essays der literarischen Moderne vorausweisen (Ralf Schnell, Tokio).

In einer Sonderveranstaltung sprach Manfred Windfuhr, Düsseldorf, als Herausgeber unter dem Titel »Rückschau und Bilanz« über das Editionsprofil der 1997 abgeschlossenen Düsseldorfer Historisch-kritischen Heine-Ausgabe. Große öffentliche Resonanz fanden auch die drei Abendvorträge im Malkasten am 26., 27. und 29. Mai 1997. Auf besonderes Interesse stieß gleich der erste Vortrag von Odo Marquard (Gießen), der Heine provokativ für eine aufgeklärte Bürgerlichkeit in Anspruch nahm und damit Diskussionen und mehrfache Rückbezüge in den Kongreßtagen initiierte. Jacques Revel (Paris) sprach über die Geschichte der deutsch-französischen Beziehungen und über Heines wichtige Rolle im Wandel der gegenseitigen Wahrnehmungen und Vorstellungen der beiden Länder. Heines Verhältnis zu Deutschland - "aus französischer Distanz, liebevoll und beunruhigt zugleich « - stand im Mittelpunkt des Vortrags von Georges-Arthur Goldschmidt (Paris), der - geprägt von biographisch-familiären Parallelen zu Heine - für kritische Skepsis gegen jede Form von Machtanspruch auch im heutigen Kontext plädierte.

Sämtliche Vorträge wurden, wie oben bereits erwähnt, von Moderatorinnen und Moderatoren begleitet, die ihrerseits für die Vortragenden, aber auch für die übrigen Teilnehmerinnen und Teilnehmer gern wahrgenommene Ansprechpartner aus der Mitte der Heine-Forschung darstellten. Wir möchten daher nicht versäumen, neben allen Vortragenden, die ihre Manuskripte zur Verfügung gestellt haben und hiermit zur erneuten und erweiterten Diskussion stellen, auch folgenden Damen und Herren von ganzem Herzen zu danken, die durch Übernahme von Moderationen wesentlich zum Erfolg des Kongresses beigetragen haben und hier in alphabetischer Folge verzeichnet sein sollen:

Marion Aptroot (Düsseldorf), Klaus Briegleb (Hamburg), Jürgen Brummack (Tübingen), Alberto Destro (Bologna), Michel Espagne (Paris), Alfred Estermann (Frankfurt/ Main), Mounir Fendri (Tunis), Karlheinz Fingerhut (Ludwigsburg), 
Elke Frederiksen (College Park/ Maryland), Wilhelm Gössmann (Düsseldorf), Volkmar Hansen (Düsseldorf), Peter Hasubek (Braunschweig), Jost Hermand (Madison/ Wisconsin), Walter Hinck (Köln), Robert C. Holub (Berkeley/ California), Hans Otto Horch (Aachen), Lothar Jordan (Osnabrück), Jocelyne Kolb (Northampton/ Massachusetts), Bernd Kortländer (Düsseldorf), Jean-Pierre Lefebvre (Paris), Ingrid Pepperle (Berlin), Ernst-Ullrich Pinkert (Aalborg), Terence James Reed (Oxford), Inge Rippmann (Basel), Jeffrey L. Sammons (New Haven/ Connecticut), Helmut Schanze (Siegen), Renate Schlesier (Paderborn), Hans-Jürgen Schrader (Genf), Peter Stein (Lüneburg), Guy Stern (Detroit/ Michigan), Shaul Willi Treidel (Haifa), Martha K. Wallach (New Britain/ Connecticut), Michael Werner (Paris), Winfried Woesler (Osnabrück), Susanne Zantop (Hanover/ New Hampshire), Edda Ziegler (München).

Weiterer Dank ist schließlich für manche organisatorische Hilfen beim Kongreß sowie bei Erstellung und Druck des Kongreßbandes abzustatten: einmal möchten wir Mechthild Niehaus für ihre organisatorische Mitarbeit beim Kongreß selbst und bei der Texterfassung für den Kongreßband unseren Dank sagen, dann Bernd Füllner für seine unermüdliche technische und wissenschaftliche Unterstützung. Auch dem Metzler-Verlag gebührt für die Betreuung eines in vieler Hinsicht so schwergewichtigen Bandes unser herzlicher Dank.

Düsseldorf, im September 1998

Karin Füllner

Joseph A. Kruse

Bernd Witte 